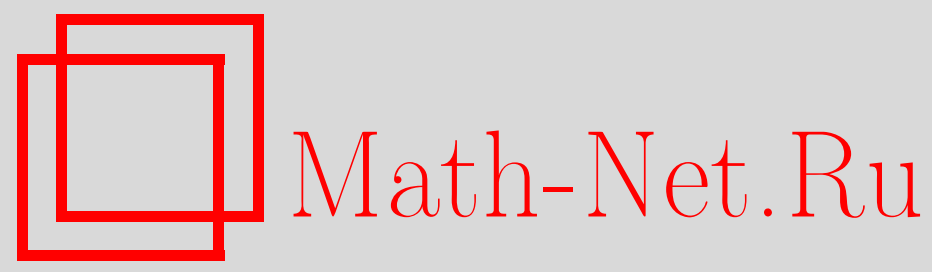

С. Н. Селезнева, О приближении с заданной точностью функций $k$-значных логик полиномами, Дискрет. матем., 2008, том 20, выпуск 2, 32-45

DOI: https://doi.org/10.4213/dm1002

Использование Общероссийского математического портала Math-Net.Ru подразумевает, что вы прочитали и согласны с пользовательским соглашением http://www . mathnet.ru/rus/agreement

Параметры загрузки:

IP: 3.85 .183 .62

26 апреля 2023 г., 14:15:31 


\title{
О приближении с заданной точностью функций $k$-значных логик полиномами
}

\author{
() 2008 г. С. Н. Селезнева
}

\begin{abstract}
Рассматриваются приближения функций $k$-значных логик полиномами с разными точностями. Находятся оценки ранга и длины приближающих полиномов в зависимости от точности приближения.

Работа выполнена при поддержке Российского фонда фундаментальных исследований, проекты 06-01-00438-а и 07-01-00444.
\end{abstract}

\section{1. Введение}

Задачи о возможностях приближения одних функций другими, более простыми в некоторых смыслах, находят применение в приложениях. В [1] рассматривались приближенные задания булевых функций стандартными формами, такими, как полиномы Жегалкина, ДНФ и некоторыми их обобщениями, и получены оценки сложности таких заданий.

В настоящей работе рассматриваются приближения с заданной точностью функций $k$-значных логик полиномами и находятся оценки ранга и длины приближающих полиномов.

\section{2. Основные понятия}

Пусть $k \geqslant 2, E_{k}=\{0,1, \ldots, k-1\}$. Функцией $k$-значной логики называется отображение $f^{n}: E_{k}^{n} \rightarrow E_{k}, n=0,1, \ldots$ Множество всех функций $k$-значной логики обозначим через $P_{k}$, множество всех функций $k$-значной логики, зависящих от переменных $x_{1}, \ldots, x_{n}$, обозначим через $P_{k}^{n}$. Для функций из $P_{k}^{n}$ наборы значений переменных множества $E_{k}^{n}$ будем называть точками.

Будем задавать функции $k$-значной логики полиномами. Под сложением и умножением будем понимать сложение и умножение по модулю $k$. Выражение вида $x_{i_{1}}^{m_{1}} \ldots x_{i_{r}}^{m_{r}}$, где $1 \leqslant m_{1}, \ldots, m_{r} \leqslant k-1$, называется мономом. Рангом монома называется число $r$. Мы будем считать константу 1 вырожденным мономом ранга 0 . Сумму вида $\sum_{i=1}^{l} c_{i} X_{i}$, где $c_{i} \in E_{k} \backslash\{0\}, X_{i}-$ различные мономы, $i=1, \ldots, l$, назовем полиномом. Длиной полинома называется число $l$. Рангом полинома назовем наибольший ранг его слагаемых. Мы будем полагать константу 0 вырожденным полиномом с рангом и длиной, равными 0 .

Пусть $k-$ простое число. В этом случае каждую функцию $k$-значной логики можно однозначно записать полиномом [2]. Обозначим полином, задающий функцию $f\left(x_{1}, \ldots, x_{n}\right)$, через $P(f)$. Ранг и длину полинома $P(f)$ обозначим $r(f)$ и $l(f)$ соответственно. 
Если $f, g \in P_{k}^{n}$, то назовем расстоянием между функциями $f$ и $g$ величину $d(f, g)$, равную числу точек из множества $E_{k}^{n}$, в которых эти функции различаются.

Пусть $\delta, 0 \leqslant \delta \leqslant 1,-$ действительное число.

Для функции $f\left(x_{1}, \ldots, x_{n}\right)$ функция $g\left(x_{1}, \ldots, x_{n}\right)$ называется приближением с точностью $\delta$, или $\delta$-приближением, если доля точек из $E_{k}^{n}$, в которых функции $f$ и $g$ отличаются, не превосходит $\delta$, то есть $d(f, g) / k^{n} \leqslant \delta$. Если при этом $\delta=q / k^{n}$, где $q$, $q \geqslant 1,-$ натуральное число, то функцию $g\left(x_{1}, \ldots, x_{n}\right)$ назовем приближением функции $f\left(x_{1}, \ldots, x_{n}\right)$ с точностью до $q$ точек. В этом случае функции $f$ и $g$ отличаются не более, чем в $q$ точках из $E_{k}^{n}$, то есть $d(f, g) \leqslant q$.

Будем исследовать числовые характеристики полиномов, которые при каждом $n$ приближают с заданной точностью все функции из $P_{k}^{n}$.

Пусть $\delta_{n}, 0 \leqslant \delta_{n} \leqslant 1, n=0,1, \ldots,-$ последовательность действительных чисел. Пусть при каждом $n$ величина $r_{k}^{\delta_{n}}(n)\left(l_{k}^{\delta_{n}}(n)\right)$ обозначает такое наименьшее число, что полиномами над переменными $x_{1}, \ldots, x_{n}$ ранга, не большего $r_{k}^{\delta_{n}}(n)$ (длины, не большей $\left.l_{k}^{\delta_{n}}(n)\right)$, можно приблизить все функции из $P_{k}^{n}$ с точностью $\delta_{n}$. Другими словами, пусть для функции $f \in P_{2}^{n}$ определены $r^{\delta}(f)=\min r(g)$ и $l^{\delta}(f)=\min l(g)$, где минимум берется по всем функциям $g \in P_{2}^{n}$, являющимся приближениями с точностью $\delta$ функции $f$. Тогда $r_{k}^{\delta_{n}}(n)=\max r^{\delta_{n}}(f)$ и $l_{k}^{\delta_{n}}(n)=\max l^{\delta_{n}}(f)$, где максимум берется по всем функциям $f$ из $P_{k}^{n}$.

В зависимости от вида последовательности $\delta_{n}$ введем некоторые упрощения обозначений. Если $\delta_{n}=\delta$, где $\delta, 0 \leqslant \delta \leqslant 1$, - действительная постоянная, то величины $r_{k}^{\delta_{n}}(n)$ и $l_{k}^{\delta_{n}}(n)$ будем обозначать $r_{k}^{\delta}(n)$ и $l_{k}^{\delta}(n)$ соответственно.

Если $\delta_{n}=q / k^{n}$, где $q \geqslant 1-$ натуральное число, то величины $r_{k}^{\delta_{n}}(n)$ и $l_{k}^{\delta_{n}}(n)$ будем обозначать $r_{k}^{(q)}(n)$ и $l_{k}^{(q)}(n)$ соответственно. Заметим, что в этом случае каждая функция $f \in P_{k}^{n}$ будет приближаться с точностью до $q$ точек.

Предложение 1. Пусть $k, k \geqslant 2$, - простое число, $\delta, 0<\delta \leqslant 1$, - действительная постоянная, $\delta_{n}, 0<\delta_{n} \leqslant 1$, - последовательность действительных чисел, стремящихся $\kappa$ нулю, $1 / k^{n}=\bar{o}\left(\delta_{n}\right)$, число q натуральное, $q \geqslant 1$.

Тогда для всех $n$, начиная с некоторого значения $n_{0}$,

(1) $0 \leqslant r_{k}^{\delta}(n) \leqslant r_{k}^{\delta_{n}}(n) \leqslant r_{k}^{(q)}(n) \leqslant \ldots \leqslant r_{k}^{(1)}(n) \leqslant n$,

(2) $0 \leqslant l_{k}^{\delta}(n) \leqslant l_{k}^{\delta_{n}}(n) \leqslant l_{k}^{(q)}(n) \leqslant \ldots \leqslant l_{k}^{(1)}(n) \leqslant k^{n}$.

В [1] были получены следующие оценки сложности приближения булевых функций:

(1) $r_{2}^{\delta}(n)=0$ при $\delta \geqslant 1 / 2$;

(2) $r_{2}^{\delta}(n) \sim(1 / 2) n$ при $0<\delta<1 / 2$ и $n \rightarrow \infty$;

(3) $l_{2}^{\delta}(n)=1$ при $\delta \geqslant 1 / 2$;

(4) $l_{2}^{\delta}(n) \leqslant((1-\delta) / 2) 2^{n}(1+\varepsilon(n))$ при $0<\delta<1 / 2$, где $\varepsilon(n) \rightarrow 0$ при $n \rightarrow \infty$.

Мы докажем следующие оценки.

Теорема 1. Пусть число $k, k \geqslant 2$, простое, $\delta, 0 \leqslant \delta \leqslant 1$, - действительная константа, $\delta_{n}, 0<\delta_{n} \leqslant 1-$ последовательность, стремящихся к нулю действительных чисел, $q$, $q \geqslant 1,-$ натуральное число. Тогда

2 Дискретная математика, т.20 №2 
(1) $r_{k}^{\delta}(n)=0$ при $\delta \geqslant(k-1) / k$;

(2) $r_{k}^{\delta}(n) \sim((k-1) / k) n$ при $0<\delta<(k-1) / k u n \rightarrow \infty$;

(3) $r_{k}^{\delta_{n}}(n) \sim((k-1) / k) n n$ nрu $\log _{k}\left(1 / \delta_{n}\right)=\bar{o}(n)$ u $n \rightarrow \infty$;

(4) $r_{k}^{(q)}(n)=n$ для всех $n$, начиная с некоторого значения $n_{0}$, при $k \geqslant 3$;

(5) $n-2 \leqslant r_{2}^{(q)}(n) \leqslant n-1$ для всех $n$, начиная с некоторого значения $n_{0}$ при $q \geqslant 2$, $r_{2}^{(1)}(n)=n-1$;

(6) $r_{k}^{\delta}(n)=n$ при $\delta=0$.

Теорема 2. Пусть число $k, k \geqslant 2$, простое, $\delta, 0 \leqslant \delta \leqslant 1$, - действительная константа, $\delta_{n}, 0<\delta_{n} \leqslant 1-$ последовательность стремящихся к нулю действительных чисел, $q$, $q \geqslant 1,-$ натуральное число. Тогда

(1) $l_{k}^{\delta}(n)=1$ при $\delta \geqslant(k-1) / k$;

(2) $l_{k}^{\delta}(n) \lesssim((k-1) / k)(1-\delta) k^{n}$ при $0<\delta<(k-1) / k$ u $n \rightarrow \infty$;

(3) $l_{k}^{\delta_{n}}(n) \sim((k-1) / k) k^{n}$ nрu $\delta_{n} \geqslant((k-1) / k)^{n} u n \rightarrow \infty$;

(4) $l_{2}^{(q)}(n) \sim(1 / 2) 2^{n}$ nри $n \rightarrow \infty$;

(5) $l_{k}^{\delta}(n)=k^{n}$ при $\delta=0$.

\section{3. О свойствах биномиальных сумм}

Вначале докажем вспомогательные леммы.

\section{1. Свойства функций $k$-значной энтропии}

Рассмотрим функцию действительного аргумента

$$
H_{k}(x)=x \log _{k} \frac{1}{x}+(1-x) \log _{k} \frac{1}{1-x}+x \log _{k}(k-1)
$$

на интервале $(0,1)$. Назовем ее функцией $k$-значной энтропии. Исследуем ее свойства.

1. Функция $H_{k}(x)$ непрерывна в своей области определения.

2. Найдем ее первую производную: $\left(H_{k}(x)\right)^{\prime}=\log _{k}((k-1)(1-x)) / x$. Отсюда получаем, что $x=(k-1) / k-$ точка максимума. Заметим, что

$$
H_{k}((k-1) / k)=1, \quad \lim _{x \rightarrow 0} H_{k}(x)=0, \quad \lim _{x \rightarrow 1} H_{k}(x)=\log _{k}(k-1) .
$$

Следовательно, при $x \in(0,(k-1) / k]$ функция $H_{k}(x)$ строго возрастает, принимая значения из полуинтервала $(0,1]$, а при $x \in[(k-1) / k, 1)$ функция $H_{k}(x)$ строго убывает, принимая значения из полуинтервала $\left[1, \log _{k}(k-1)\right)$.

3. Найдем ее вторую производную: $\left.\left(H_{k}(x)\right)^{\prime \prime}=1 /(x(x-1)) \ln k\right)$. В области определения функции $H_{k}(x)$ ее вторая производная отрицательна. Следовательно, функция $H_{k}(x)$ во всей области определения выпукла вверх. 


\section{2. Свойства биномиальных сумм}

Лемма 1. Для всех натуральных $n, n \geqslant 1, u r \leqslant n$

$$
\left(\begin{array}{l}
n \\
r
\end{array}\right) \leqslant \frac{n^{n}}{r^{r}(n-r)^{n-r}}
$$

(для определенности полагаем, что $0^{0}=1$ ). При этом, если $r=\lambda n$ для некоторого $\lambda$, $0<\lambda<1$, mо

$$
\left(\begin{array}{l}
n \\
r
\end{array}\right) \leqslant(\lambda)^{-\lambda n}(1-\lambda)^{-(1-\lambda) n}
$$

Доказательство. Доказательство проведем индукцией по значению $n$.

В качестве базиса индукции возьмем следующий случай. Заметим, что для всех $n \geqslant 1$ справедливы равенства $\left(\begin{array}{l}n \\ 0\end{array}\right)=\left(\begin{array}{l}n \\ n\end{array}\right)=1$ и утверждение леммы 1 для $r=0$ и $r=n$ выполняется.

Проведем индуктивный переход. Пусть для всех натуральных $n^{\prime}, n^{\prime}<n$, и $r \leqslant n^{\prime}$ первое утверждение леммы 1 верно. Рассматривая $\left(\begin{array}{l}n \\ r\end{array}\right)$ при $1 \leqslant r \leqslant n$, получаем, что

$$
\left(\begin{array}{l}
n \\
r
\end{array}\right)=\frac{n !}{r !(n-r) !}=\frac{(n-1) !}{(r-1) !(n-r) !} \frac{n}{r}=\left(\begin{array}{c}
n-1 \\
r-1
\end{array}\right) \frac{n}{r} .
$$

По предположению индукции

$$
\left(\begin{array}{l}
n-1 \\
r-1
\end{array}\right) \leqslant \frac{(n-1)^{n-1}}{(r-1)^{r-1}(n-r)^{n-r}}
$$

Поэтому

$$
\begin{aligned}
\left(\begin{array}{l}
n \\
r
\end{array}\right) & \leqslant \frac{(n-1)^{n-1}}{(r-1)^{r-1}(n-r)^{n-r}} \frac{n}{r}=\frac{(n-1)^{n-1}}{(r-1)^{r-1}(n-r)^{n-r}} \frac{n^{n-1}}{n^{n-1}} \frac{r^{r-1}}{r^{r-1}} \frac{n}{r} \\
& =\frac{(1+1 /(r-1))^{r-1}}{(1+1 /(n-1))^{n-1}} \frac{n^{n}}{r^{r}(n-r)^{n-r}} \leqslant \frac{n^{n}}{r^{r}(n-r)^{n-r}} .
\end{aligned}
$$

Здесь мы воспользовались тем, что последовательность $(1+1 / n)^{n}$ является неубывающей.

Пусть теперь $r=\lambda n$, где $0<\lambda<1$. Тогда

$$
\left(\begin{array}{l}
n \\
r
\end{array}\right)=\left(\begin{array}{c}
n \\
\lambda n
\end{array}\right) \leqslant \frac{n^{n}}{(\lambda n)^{\lambda n}((1-\lambda) n)^{(1-\lambda) n}}=\lambda^{-\lambda n}(1-\lambda)^{-(1-\lambda) n} .
$$

Лемма 1 доказана.

Лемма 2. Пусть $k \geqslant 2 u r<k-1 n / k$. Тогда

$$
\sum_{i=0}^{r}\left(\begin{array}{l}
n \\
i
\end{array}\right)(k-1)^{i} \leqslant \frac{(k-1)(n-r)}{(k-1) n-k r}\left(\begin{array}{l}
n \\
r
\end{array}\right)(k-1)^{r} .
$$


Если при этом $\lambda=r / n \leqslant \delta$ для некоторого действительного числа $\delta<(k-1) / k$, то

$$
\sum_{i=0}^{r}\left(\begin{array}{l}
n \\
i
\end{array}\right)(k-1)^{i} \leqslant \frac{1}{1-k \delta /(k-1)} k^{H_{k}(\delta) n} .
$$

Пусть $k \geqslant 2$ u $r>(k-1) n / k$. Тогда

$$
\sum_{i=r}^{n}\left(\begin{array}{l}
n \\
i
\end{array}\right)(k-1)^{i} \leqslant \frac{r}{k r-(k-1) n}\left(\begin{array}{l}
n \\
r
\end{array}\right)(k-1)^{r} .
$$

Если при этом $1-\lambda=r / n \geqslant 1-\delta$ для некоторого действительного числа $\delta<1 / k$, то

$$
\sum_{i=0}^{r}\left(\begin{array}{l}
n \\
i
\end{array}\right)(k-1)^{i} \leqslant \frac{1}{1-k \delta} k^{H_{k}(\delta) n} .
$$

Доказательство. Докажем первое утверждение. Пусть $r<(k-1) / k n$. Следуя рассуждениям из [2], подсчитаем сумму $\sum_{i=0}^{r}\left(\begin{array}{c}n \\ i\end{array}\right)(k-1)^{i}$. Нетрудно видеть, что

$$
\begin{aligned}
\sum_{i=0}^{r}\left(\begin{array}{l}
n \\
i
\end{array}\right)(k-1)^{i} & =\left(\begin{array}{l}
n \\
0
\end{array}\right)(k-1)^{0}+\left(\begin{array}{l}
n \\
1
\end{array}\right)(k-1)^{1}+\ldots+\left(\begin{array}{l}
n \\
r
\end{array}\right)(k-1)^{r} \\
& =\left(\begin{array}{l}
n \\
r
\end{array}\right)(k-1)^{r}+\ldots+\left(\begin{array}{l}
n \\
1
\end{array}\right)(k-1)^{1}+\left(\begin{array}{l}
n \\
0
\end{array}\right)(k-1)^{0} \\
& <\left(\begin{array}{l}
n \\
r
\end{array}\right)(k-1)^{r}\left(1+\frac{r}{(n-r+1)(k-1)}+\frac{r^{2}}{(n-r+1)^{2}(k-1)^{2}}+\ldots\right) \\
& <\left(\begin{array}{l}
n \\
r
\end{array}\right)(k-1)^{r}\left(1+\frac{r}{(n-r)(k-1)}+\frac{r^{2}}{(n-r)^{2}(k-1)^{2}}+\ldots\right) .
\end{aligned}
$$

При $r<(k-1) n / k$ справедливо неравенство $r /((n-r)(k-1))<1$, то есть в скобках стоит сумма бесконечно убывающей геометрической прогрессии. Она равна

$$
\frac{1}{1-r /((n-r)(k-1))}=\frac{(k-1)(n-r)}{(k-1) n-k r} \text {. }
$$

Пусть теперь $r=\lambda n \leqslant \delta n$ для некоторого действительного числа $\delta<(k-1) / k$. Тогда, используя лемму 1 и свойства функции $H_{k}(x)$, получаем, что

$$
\begin{aligned}
\left(\begin{array}{l}
n \\
r
\end{array}\right)(k-1)^{r} & =\left(\begin{array}{c}
n \\
\lambda n
\end{array}\right)(k-1)^{\lambda n} \\
& \leqslant \lambda^{-\lambda n}(1-\lambda)^{-(1-\lambda) n}(k-1)^{\lambda n}=k^{H_{k}(\lambda) n} \leqslant k^{H_{k}(\delta) n} .
\end{aligned}
$$

Отсюда получаем

$$
\begin{aligned}
\sum_{i=0}^{r}\left(\begin{array}{l}
n \\
i
\end{array}\right)(k-1)^{i} & \leqslant \frac{(k-1)(n-r)}{(k-1) n-k r}\left(\begin{array}{l}
n \\
r
\end{array}\right)(k-1)^{r} \\
& =\frac{1-\lambda}{1-k \lambda /(k-1)}\left(\begin{array}{l}
n \\
r
\end{array}\right)(k-1)^{r} \leqslant \frac{1}{1-k \delta /(k-1)} k^{H_{k}(\delta) n} .
\end{aligned}
$$


Докажем второе утверждение леммы. Пусть $r>(k-1) n / k$. Подсчитаем сумму $\sum_{i=r}^{n}\left(\begin{array}{c}n \\ i\end{array}\right)(k-1)^{i}$. Нетрудно видеть, что

$$
\begin{aligned}
\sum_{i=r}^{n}\left(\begin{array}{l}
n \\
i
\end{array}\right)(k-1)^{i} & =\left(\begin{array}{l}
n \\
r
\end{array}\right)(k-1)^{r}+\left(\begin{array}{c}
n \\
r+1
\end{array}\right)(k-1)^{r+1}+\cdots+\left(\begin{array}{l}
n \\
n
\end{array}\right)(k-1)^{n} \\
& <\left(\begin{array}{l}
n \\
r
\end{array}\right)(k-1)^{r}\left(1+(k-1) \frac{n-r}{r+1}+(k-1)^{2} \frac{n-r}{r+1}+\ldots\right) \\
& <\left(\begin{array}{l}
n \\
r
\end{array}\right)(k-1)^{r}\left(1+(k-1) \frac{n-r}{r}+(k-1)^{2} \frac{n-r}{r}+\ldots\right) \\
& <\left(\begin{array}{c}
n \\
r
\end{array}\right)(k-1)^{r} \frac{1}{1-(k-1)(n-r) / r}=\left(\begin{array}{c}
n \\
r
\end{array}\right)(k-1)^{r} \frac{r}{k r-(k-1) n}
\end{aligned}
$$

Здесь было учтено, что при $r>(k-1) n / k$ справедливо неравенство $(n-r) / r<1$ и в скобках стоит сумма бесконечно убывающей геометрической прогрессии.

Пусть теперь $r=(1-\lambda) n \geqslant(1-\delta) n$ для некоторого действительного числа $\delta<1 / k$. Тогда, используя лемму 1 и свойства функции $H_{k}(x)$, получаем, что

$$
\begin{aligned}
\left(\begin{array}{l}
n \\
r
\end{array}\right)(k-1)^{r} & =\left(\begin{array}{c}
n \\
\lambda n
\end{array}\right)(k-1)^{\lambda n} \\
& \leqslant \lambda^{-\lambda n}(1-\lambda)^{-(1-\lambda) n}(k-1)^{\lambda n}=k^{\left.H_{k}(1-\lambda)\right) n} \leqslant k^{H_{k}(1-\delta) n} .
\end{aligned}
$$

Отсюда следует, что

$$
\begin{aligned}
\sum_{i=r}^{n}\left(\begin{array}{l}
n \\
i
\end{array}\right)(k-1)^{i} & \leqslant \frac{r}{k r-(k-1) n}\left(\begin{array}{l}
n \\
r
\end{array}\right)(k-1)^{r} \\
& =\frac{1-\lambda}{1-k \lambda}\left(\begin{array}{l}
n \\
r
\end{array}\right)(k-1)^{r} \leqslant \frac{1}{1-k \delta} k^{H_{k}(1-\delta) n} .
\end{aligned}
$$

Лемма 2 доказана.

Лемма 3. Пусть $k \geqslant 2$ и $r(n)$ - последовательность натуральных чисел,

$$
\lambda_{n}=\frac{r(n)}{n} \leqslant \delta<\frac{k-1}{k}
$$

где $\delta$ - действительная константа. Тогда существует такая действительная констанma $\varepsilon, 0<\varepsilon<1$, чmо

$$
\sum_{i=0}^{r(n)}\left(\begin{array}{l}
n \\
i
\end{array}\right)(k-1)^{i} \leqslant k^{\varepsilon n}
$$

для всех $n$, начиная с некоторого значения $n_{0}$.

Доказательство. По лемме 2

$$
\sum_{i=0}^{r(n)}\left(\begin{array}{l}
n \\
i
\end{array}\right)(k-1)^{i}=\sum_{i=0}^{\lambda_{n} n}\left(\begin{array}{l}
n \\
i
\end{array}\right)(k-1)^{i} \leqslant \frac{1}{1-k \delta /(k-1)} k^{H_{k}(\delta) n} .
$$


Отсюда, учитывая, что $H_{k}(\delta)<H_{k}((k-1) / k)=1$, получаем, что существует такая действительная константа $\varepsilon, 0<\varepsilon<1$, что

$$
\sum_{i=0}^{r(n)}\left(\begin{array}{l}
n \\
i
\end{array}\right)(k-1)^{i} \leqslant k^{\varepsilon n}
$$

для всех $n$, начиная с некоторого значения $n_{0}$. Лемма 3 доказана.

Лемма 4. Пусть $k \geqslant 2$ и $r(n)$ - последовательность натуральньх чисел,

$$
\lambda_{n}=\frac{r(n)}{n} \leqslant \delta_{n},
$$

где $\delta_{n}$ - последовательность стремящихся к нулю положительных действительных чисел. Тогда для любой действительной константы $\varepsilon>0$ существует такая действительная константа $\varepsilon_{1}, 0<\varepsilon_{1}<1$, что для любой последовательности $l(n)$ положительных чисел такой, что $l(n) \leqslant((k-1) / k-\varepsilon) n$,

$$
\sum_{i=0}^{r(n)}\left(\begin{array}{l}
n \\
i
\end{array}\right)(k-1)^{i} \sum_{j=0}^{l(n)}\left(\begin{array}{l}
n \\
j
\end{array}\right)(k-1)^{j} \leqslant k^{\left(1-\varepsilon_{1}\right) n}
$$

для всех $n$, начиная с некоторого значения $n_{0}$.

Доказательство. При каждом $n$ рассмотрим произведение

$$
\sum_{i=0}^{r(n)}\left(\begin{array}{l}
n \\
i
\end{array}\right)(k-1)^{i} \sum_{j=0}^{l(n)}\left(\begin{array}{l}
n \\
j
\end{array}\right)(k-1)^{j} .
$$

По лемме 2 выписанное произведение не больше

$$
\frac{1}{1-k \delta_{n}} /(k-1) k^{H_{k}\left(\delta_{n}\right) n} \frac{1}{1-k((k-1) / k-\varepsilon) /(k-1)} k^{H_{k}((k-1) / k-\varepsilon) n}
$$

для всех $n$, начиная с некоторого значения $n_{0}^{\prime}$.

Оценим $H_{k}\left(\delta_{n}\right)+H_{k}((k-1) / k-\varepsilon)$. Для этого рассмотрим функцию

$$
H_{k}^{\prime}(x)=H_{k}(x)+H_{k}((k-1) / k-\varepsilon)
$$

на интервале $(0,(k-1) / k)$. Найдем ее свойства. Нетрудно видеть, что

(1) $\lim _{x \rightarrow 0} H_{k}^{\prime}(x)=H_{k}((k-1) / k-\varepsilon)<1$,

(2) функция $H_{k}^{\prime}(x)$ непрерывна на промежутке $(0,(k-1) / k)$.

Следовательно, найдется такая окрестность $I$ справа от нуля и такое действительное число $\varepsilon_{2}, 0<\varepsilon_{2}<1$, что для всех $x, x \in I$, справедливо неравенство $H_{k}^{\prime}(x) \leqslant 1-\varepsilon_{2}$.

Если $\delta_{n}-$ последовательность положительных чисел и $\delta_{n} \rightarrow 0$ при $n \rightarrow \infty$, то для всех $n$, начиная с некоторого значения $n_{0}^{\prime \prime}$, справедливо включение $\delta_{n} \in I$, и значит, $H_{k}^{\prime}\left(\delta_{n}\right) \leqslant 1-\varepsilon_{2}$. Другими словами

$$
\sum_{i=0}^{r(n)}\left(\begin{array}{l}
n \\
i
\end{array}\right)(k-1)^{i} \sum_{j=0}^{l(n)}\left(\begin{array}{l}
n \\
j
\end{array}\right)(k-1)^{j} \leqslant C k^{\left(1-\varepsilon_{2}\right) n} \leqslant k^{\left(1-\varepsilon_{1}\right) n}
$$

для некоторой константы $C>0$, некоторого действительного числа $\varepsilon_{1}, 0<\varepsilon_{1}<\varepsilon_{2}$, и всех $n$, начиная с некоторого значения $n_{0}$. Лемма 4 доказана. 


\section{4. О свойствах полиномов функций $k$-значных логик}

Пусть $X^{n}-$ множество всех мономов вида $x_{i_{1}}^{m_{1}} \ldots x_{i_{r}}^{m_{r}}$, где $1 \leqslant m_{1}, \ldots, m_{r} \leqslant k-1$, $1 \leqslant r \leqslant n, 1 \in X^{n}$.

Будем говорить, что моном $X=x_{i_{1}}^{m_{1}} \ldots x_{i_{r}}^{m_{r}}, X \in X^{n}$, и набор $\alpha=\left(a_{1}, \ldots, a_{n}\right)$, $\alpha \in E_{k}^{n}$, в котором $a_{i_{1}}=m_{1}, \ldots, a_{i_{r}}=m_{r}, a_{j}=0$ в остальных случаях, соответствуют друг другу. Константе 1 соответствует нулевой набор $0=(0, \ldots, 0)$. Заметим, что такое соответствие множеств $X^{n}$ и $E_{k}^{n}$ является взаимно однозначным.

Для монома $X$ из $X^{n}$ определим его тип $t(X)$ как множество индексов входящих в него переменных.

Для набора $\alpha=\left(a_{1}, \ldots, a_{n}\right)$ из $E_{k}^{n}$ назовем его типом $t(\alpha)$ множество индексов его ненулевых координат, его рангом $|\alpha|$ число его ненулевых координат.

Заметим, что типы соответствующих друг другу монома и набора совпадают.

Распространим понятие типа на полиномы и множества наборов. Пусть

$$
p\left(x_{1}, \ldots, x_{n}\right)=\sum_{i=1}^{l} c_{i} X_{i}
$$

- полином, множеством типов его слагаемых назовем множество

$$
t(p)=\bigcup_{i=1}^{l} t\left(X_{i}\right) .
$$

По определению положим $t(0)=\varnothing$. Пусть $E \subseteq E_{k}^{n}$, множеством типов его элементов называется множество

$$
t(E)=\bigcup_{\alpha \in E} t(\alpha) .
$$

Лемма 5. Пусть для некоторого типа $t$ функция $f\left(x_{1}, \ldots, x_{n}\right)$ из $P_{k}^{n}$ равна нулю на всех таких наборах $\alpha$, что $t(\alpha) \subseteq t$. Тогда полином $f\left(x_{1}, \ldots, x_{n}\right)$ не содержит таких слагаемых $X$, что $t(X) \subseteq t$.

Доказательство. Пусть $t=\left\{i_{1}, \ldots, i_{r}\right\}$. Предположим противное: пусть полином $f\left(x_{1}, \ldots, x_{n}\right)$ содержит такое слагаемое $X$, что $t(X) \subseteq t$.

Для полинома $f\left(x_{1}, \ldots, x_{n}\right)$ положим $x_{j}=0$ для всех $j \neq i_{1}, \ldots, i_{r}$, получим некоторый полином $g\left(x_{i_{1}}, \ldots, x_{i_{r}}\right)$. Заметим, что $g-$ не пустой полином, так как по меньшей мере он содержит слагаемое $X$. Так как каждая функция $k$-значной логики при простом $k$ задается полиномом однозначно и тождественно равную нулю функцию задает пустой полином (см. [2]), функция $g\left(x_{i_{1}}, \ldots, x_{i_{r}}\right)$ принимает ненулевое значение хотя бы на одном наборе значений переменных. Приходим к противоречию, так как по описанию функции $f\left(x_{1}, \ldots, x_{n}\right)$ получающаяся из нее функция $g\left(x_{i_{1}}, \ldots, x_{i_{r}}\right)$ тождественно равна нулю. Следовательно, предположение неверно и полином $f\left(x_{1}, \ldots, x_{n}\right)$ не содержит таких слагаемых $X$, что $t(X) \subseteq t$. Лемма 5 доказана.

Следствие 1. Если функция $f\left(x_{1}, \ldots, x_{n}\right)$ из $P_{k}^{n}$ равна нулю на всех наборах ранга, не большего $r$, по полином $f\left(x_{1}, \ldots, x_{n}\right)$ не содержит слагаемых ранга, не превосходящего $r$.

Пусть $E \subseteq E_{k}^{n}$. Назовем множество $E$ стабильным, если оно обладает следующим свойством: если $E$ содержит набор типа $t$, то оно содержит также все возможные наборы типа $t$. 
Лемма 6. Пусть $E \subseteq E_{k}^{n}$, и пусть множество $E$ стабильно. Тогда для любой функции $f\left(x_{1}, \ldots, x_{n}\right)$ из $P_{k}^{n}$ найдется совпадающий с функцией $f\left(x_{1}, \ldots, x_{n}\right)$ на множестве $E$ полином $g\left(x_{1}, \ldots, x_{n}\right)$, для которого $t(g) \subseteq t(E)$.

Доказательство. Доказательство проведем индукцией по числу различных типов в множестве $E$.

Найдем базис индукции. Пусть множество $E$ содержит наборы только одного типа $t$. Пусть $t=\left\{i_{1}, \ldots, i_{r}\right\}$. Рассмотрим функцию

$$
f^{\prime}\left(x_{1}, \ldots, x_{n}\right)= \begin{cases}f\left(x_{1}, \ldots, x_{n}\right) & \text { на } E, \\ 0 & \text { на } E_{k}^{n} \backslash E .\end{cases}
$$

Положив в $f^{\prime}\left(x_{1}, \ldots, x_{n}\right)$ равными нулю все переменные $x_{j}$ при $j \neq i_{1}, \ldots, i_{r}$, получим функцию $g^{\prime}\left(x_{i_{1}}, \ldots, x_{i_{r}}\right)$. По следствию 1 полином $g^{\prime}\left(x_{i_{1}}, \ldots, x_{i_{r}}\right)$ может содержать только слагаемые ранга $r$. Следовательно, $t\left(g^{\prime}\right) \subseteq t(E)$.

По построению значения полинома $g\left(x_{1}, \ldots, x_{n}\right)=g^{\prime}\left(x_{i_{1}}, \ldots, x_{i_{r}}\right)$ совпадают с значениями функции $f\left(x_{1}, \ldots, x_{n}\right)$ на множестве $E$ и $t(g) \subseteq t(E)$, таким образом, $g\left(x_{1}, \ldots, x_{n}\right)$ - искомый полином.

Проведем индуктивный переход. Пусть для каждого стабильного подмножества $E_{k}^{n}$ с числом различных типов $\tau$ утверждение леммы 6 верно. Рассмотрим стабильное множество $E, E \subseteq E_{k}^{n}$, в котором $\tau+1$ различный тип. Выделим в множестве $E$ некоторый тип $t$, $t=\left\{i_{1}, \ldots, i_{r}\right\}$, максимальный (в частичном упорядочении множества всех подмножеств из $\{1, \ldots, n\}$ по включению) среди всех типов множества $E$ (если максимальных типов несколько, можно взять любой из них).

Разобьем множество $E$ на две части: стабильное подмножество $E_{1}$, содержащее наборы всех типов, кроме типа $t$, и стабильное подмножество $E_{2}, E_{2}=E \backslash E_{1}$.

По предположению индукции найдется полином $g_{1}\left(x_{1}, \ldots, x_{n}\right)$, совпадающий с функцией $f\left(x_{1}, \ldots, x_{n}\right)$ на множестве $E_{1}$ и для которого $t\left(g_{1}\right) \subseteq t\left(E_{1}\right)$.

Рассмотрим функцию

$$
f_{2}\left(x_{1}, \ldots, x_{n}\right)= \begin{cases}f\left(x_{1}, \ldots, x_{n}\right)-g_{1}\left(x_{1}, \ldots, x_{n}\right) & \text { на } E_{2}, \\ 0 & \text { на } E_{k}^{n} \backslash E_{2} .\end{cases}
$$

По следствию 1 полином $f_{2}\left(x_{1}, \ldots, x_{n}\right)$ не содержит слагаемые ранга, меньшего $r$. Пусть $g_{2}\left(x_{1}, \ldots, x_{n}\right)$ - полином, получающийся из полинома $f_{2}\left(x_{1}, \ldots, x_{n}\right)$, если оставить в нем только слагаемые типа $t$. Заметим, что функция $g_{2}\left(x_{1}, \ldots, x_{n}\right)$ равна нулю на всех наборах веса меньшего $r$ и на наборах веса, равного $r$, но тип которых не есть $t$.

Рассмотрим полином $g\left(x_{1}, \ldots, x_{n}\right)=g_{1}\left(x_{1}, \ldots, x_{n}\right)+g_{2}\left(x_{1}, \ldots, x_{n}\right)$. Ясно, что

$$
\begin{aligned}
g\left(x_{1}, \ldots, x_{n}\right) & = \begin{cases}g_{1}\left(x_{1}, \ldots, x_{n}\right) & \text { на } E_{1}, \\
g_{1}\left(x_{1}, \ldots, x_{n}\right)+g_{2}\left(x_{1}, \ldots, x_{n}\right) & \text { на } E_{2},\end{cases} \\
& = \begin{cases}g_{1}\left(x_{1}, \ldots, x_{n}\right) & \text { на } E_{1}, \\
g_{1}\left(x_{1}, \ldots, x_{n}\right)+f\left(x_{1}, \ldots, x_{n}\right)-g_{1}\left(x_{1}, \ldots, x_{n}\right) & \text { на } E_{2},\end{cases} \\
& = \begin{cases}f\left(x_{1}, \ldots, x_{n}\right) & \text { на } E_{1}, \\
f\left(x_{1}, \ldots, x_{n}\right) & \text { на } E_{2} .\end{cases}
\end{aligned}
$$

По построению полинома $g\left(x_{1}, \ldots, x_{n}\right)$ он состоит только из слагаемых типов элементов множества $E$. Лемма 6 доказана. 
Следствие 2. Пусть $E \subseteq E_{k}^{n}$ и множество $E$ стабильно. Тогда для любой функции $f\left(x_{1}, \ldots, x_{n}\right)$ из $P_{k}^{n}$ найдется полином $g\left(x_{1}, \ldots, x_{n}\right)$, совпадающий с $f\left(x_{1}, \ldots, x_{n}\right)$ на множестве $E$, длина которого не больше $|E|$.

Следствие 3. При $0 \leqslant \delta \leqslant 1 u k \geqslant 2$

$$
l_{k}^{\delta}(n) \leqslant(1-\delta) k^{n}+(k-1)^{n} .
$$

Доказательство. Выберем стабильное множество $E, E \subseteq E_{k}^{n}$, по следующим правилам:

(1) если мы добавили некоторый набор $\alpha$ к множеству, то мы добавим к нему все наборы типа $t(\alpha)$;

(2) если в множестве меньше $\left\lceil(1-\delta) k^{n}\right\rceil$ наборов, то продолжаем добавлять к нему наборы.

Таким образом строим стабильное множество $E$, содержащее $l$ наборов. Заметим, что $\left\lceil(1-\delta) k^{n}\right\rceil \leqslant l \leqslant\left\lceil(1-\delta) k^{n}\right\rceil+(k-1)^{n}-1$. По лемме 6 найдется полином $g\left(x_{1}, \ldots, x_{n}\right)$, совпадающий с функцией $f\left(x_{1}, \ldots, x_{n}\right)$ на множестве $E$, то есть функции $f\left(x_{1}, \ldots, x_{n}\right)$ и $g\left(x_{1}, \ldots, x_{n}\right)$ различаются не более, чем в $k^{n}-\left\lceil(1-\delta) k^{n}\right\rceil \leqslant k^{n}-(1-\delta) k^{n} \leqslant \delta k^{n}$ точках, или функция $g\left(x_{1}, \ldots, x_{n}\right)$ является приближением функции $f\left(x_{1}, \ldots, x_{n}\right)$ с точностью $\delta$.

Также по следствию 2 длина полинома $g\left(x_{1}, \ldots, x_{n}\right)$ не больше $l$, то есть не больше $\left\lceil(1-\delta) k^{n}\right\rceil+(k-1)^{n}-1 \leqslant(1-\delta) k^{n}+(k-1)^{n}$. Следствие 3 доказано.

\section{5. Доказательство основных теорем}

Теперь мы можем доказать сформулированные ранее теоремы.

Доказательство теоремь 1. Докажем первое утверждение. Если $\delta \geqslant(k-1) / k$, то полином с требуемым приближением можно построить следующим образом. Найдем такое значение $c$, что число точек, в которых функция $f\left(x_{1}, \ldots, x_{n}\right)$ принимает значение $c$, не меньше, чем $(1 / k) k^{n}$. Такое значение $c$ всегда существует. Тогда приблизим функцию $f\left(x_{1}, \ldots, x_{n}\right)$ полиномом $g\left(x_{1}, \ldots, x_{n}\right)=c$. Видно, что функция $g\left(x_{1}, \ldots, x_{n}\right)$ является $\delta$-приближением функции $f\left(x_{1}, \ldots, x_{n}\right)$. Ранг полинома $g\left(x_{1}, \ldots, x_{n}\right)$ равен 0. Очевидно, что полиномом меньшего ранга при приближении функций не обойтись.

Следовательно, при $\delta \geqslant(k-1) / k$ справедливо равенство $r_{k}^{\delta}(n)=0$.

Докажем второе утверждение. Пусть $0<\delta<(k-1) / k$. Найдем верхнюю оценку. Пусть $\varepsilon-$ положительная константа и $r(n)$ - такая последовательность целых чисел, что $r(n) \leqslant((k-1) / k+\varepsilon) n$. Отсечем в полиноме функции $f\left(x_{1}, \ldots, x_{n}\right)$ все слагаемые ранга, большего $r(n)$. Получим некоторый полином, который мы обозначим $g\left(x_{1}, \ldots, x_{n}\right)$. Заметим, что функции $f\left(x_{1}, \ldots, x_{n}\right)$ и $g\left(x_{1}, \ldots, x_{n}\right)$ совпадают на всех наборах значений переменных, в которых не менее $(n-r(n))$ нулей. На оставшихся наборах эти функции могут отличаться. Наборов, в которых они могут отличаться, не больше, чем $\sum_{i=r(n)}^{n}\left(\begin{array}{c}n \\ i\end{array}\right)(k-1)^{i}$. По лемме 2

$$
\sum_{i=r(n)}^{n}\left(\begin{array}{l}
n \\
i
\end{array}\right)(k-1)^{i} \leqslant \frac{1}{k \varepsilon} k^{H_{k}((k-1) / k-\varepsilon) n} .
$$

Так как $H_{k}((k-1) / k-\varepsilon)<1$, найдется такое значение $n_{0}$, что для всех $n \geqslant n_{0}$ выполняется неравенство $1 /(k \varepsilon) k^{H_{k}((k-1) / k-\varepsilon) n} \leqslant \delta k^{n}$, то есть функция $g\left(x_{1}, \ldots, x_{n}\right)$ является приближением функции $f\left(x_{1}, \ldots, x_{n}\right)$ с точностью $\delta$. 
Найдем нижнюю оценку. Пусть $\varepsilon$ - положительная константа и $r(n)-$ такая последовательность целых чисел, что $r(n) \leqslant((k-1) / k-\varepsilon) n$. Подсчитаем при каждом $n$, сколько функций можно приблизить полиномами ранга, не большего $r(n)$.

Полиномов ранга, не большего $r(n)$, не более, чем $k^{\sum_{j=0}^{r(n)}\left(\begin{array}{c}n \\ j\end{array}\right)(k-1)^{j}}$. Каждый из них может являться $\delta$-приближением не более $\sum_{i=0}^{\left[\delta k^{n}\right]}\left(\begin{array}{c}k^{n} \\ i\end{array}\right)(k-1)^{i}$ функций, где $\left[\delta k^{n}\right]$ обозначает целую часть числа $\delta k^{n}$. Поэтому полиномами ранга, не большего $r(n)$, можно приблизить не более $k^{\sum_{j=0}^{r(n)}\left(\begin{array}{c}n \\ j\end{array}\right)(k-1)^{j}} \sum_{i=0}^{\left[\delta k^{n}\right]}\left(\begin{array}{c}k^{n} \\ i\end{array}\right)(k-1)^{i}$ функций.

По лемме 3 найдутся такие действительные константы $\varepsilon_{1}$ и $\varepsilon_{2}, 0<\varepsilon_{1}, \varepsilon_{2}<1$, что

$$
\sum_{j=0}^{r(n)}\left(\begin{array}{l}
n \\
j
\end{array}\right)(k-1)^{j} \leqslant k^{\varepsilon_{1} n}, \quad \sum_{i=0}^{\left[\delta k^{n}\right]}\left(\begin{array}{c}
k^{n} \\
i
\end{array}\right)(k-1)^{i} \leqslant k^{\varepsilon_{2} k^{n}}
$$

для всех $n$, начиная с некоторого значения $n_{0}$. Следовательно, для всех $n$, начиная со значения $n_{0}$, полиномов ранга, не большего $r(n)$, не хватает для приближения всех $k^{k^{n}}$ функций, то есть для любого $\varepsilon>0$ для всех $n$, начиная с некоторого значения $n_{0}=n_{0}(\varepsilon)$,

$$
r_{k}^{\delta}(n) \geqslant((k-1) / k-\varepsilon) n .
$$

Собирая вместе верхнюю и нижнюю оценки, получаем, что $r_{k}^{\delta}(n) \sim n(k-1) / k$ при $n \rightarrow \infty$.

Докажем третье утверждение. Пусть $\delta_{n}, 0<\delta_{n} \leqslant 1$, - стремящаяся к нулю последовательность действительных чисел и $1 / k^{n}=\bar{o}\left(\delta_{n}\right)$ при $n \rightarrow \infty$.

Верхняя оценка доказывается аналогично доказательству верхней оценки в утверждении 2 теоремы 1, так как оценка

$$
\frac{1}{k \varepsilon} k^{H_{k}((k-1) / k-\varepsilon) n} \leqslant \delta_{n} k^{n}
$$

будет выполняться для всех $n$, начиная с некоторого значения $n_{0}$, при указанных ограничениях на последовательность $\delta_{n}$.

Нижняя оценка следует из предложения 1 и утверждения 2 теоремы 1, так как

$$
r_{k}^{\delta_{n}}(n) \geqslant r_{k}^{\delta}(n) \geqslant((k-1) / k-\varepsilon) n
$$

для любого $\varepsilon>0$. Поэтому $r_{k}^{\delta_{n}} \sim n(k-1) / k$ при $n \rightarrow \infty$.

Докажем четвертое утверждение. Рассмотрим функцию $f\left(x_{1}, \ldots, x_{n}\right)$ из $P_{k}^{n}$ при $k \geqslant 3$. По предложению 1 справедливо неравенство $r_{k}^{(q)}(n) \leqslant n$. Подсчитаем, сколько функций можно приблизить полиномами ранга, не большего $n-1$. Всего таких полиномов не более $k^{k^{n}-(k-1)^{n}}$. Каждый из них может приближать не более $\sum_{i=0}^{q}\left(\begin{array}{c}k^{n} \\ i\end{array}\right) \leqslant q k^{q n}$ функций. Следовательно, всего полиномами ранга, не большего $n-1$, можно приблизить не больше $k^{k^{n}-(k-1)^{n}} q k^{q n}$ функций. Последнее выражение меньше $k^{k^{n}}$ при $k \geqslant 3$ для всех $n$, начиная с некоторого $n_{0}$, то есть $r_{k}^{(q)}(n) \geqslant n$ для всех $n$, начиная с некоторого $n_{0}$, при $k \geqslant 3$. Следовательно, $r_{k}^{(q)}(n)=n$ для всех $n$, начиная с некоторого значения $n_{0}$, при $k \geqslant 3$.

Докажем пятое утверждение. Рассмотрим функцию $f\left(x_{1}, \ldots, x_{n}\right)$ из $P_{2}^{n}$. Пусть полином $g\left(x_{1}, \ldots, x_{n}\right)$ получен из полинома $f\left(x_{1}, \ldots, x_{n}\right)$ вычеркиванием монома ранга $n$ (если он есть). Тогда значения функций $f\left(x_{1}, \ldots, x_{n}\right)$ и $g\left(x_{1}, \ldots, x_{n}\right)$ отличаются не более, чем в одной точке. Следовательно, $r_{2}^{(q)}(n) \leqslant r_{2}^{(1)}(n) \leqslant n-1$. 
С другой стороны, подсчитаем, сколько функций можно приблизить полиномами ранга, не большего $n-3$. Всего таких функций не более $2^{2^{n}-\left(\begin{array}{c}n \\ 2\end{array}\right)-n-1}$. Каждая из них может приближать не более $\sum_{i=0}^{q}\left(\begin{array}{c}2^{n} \\ i\end{array}\right) \leqslant q 2^{q n}$ функций. Следовательно, всего полиномами ранга, не большего $n-3$, можно приблизить не более $2^{2^{n}-n(n-1) / 2-n-1} q 2^{q n}$ функций. Последнее выражение меньше $2^{2^{n}}$ для всех $n$, начиная с некоторого значения $n_{0}$, то есть $r_{2}^{(q)}(n) \geqslant n-2$ при $q \geqslant 2$.

Если $q=1$, то подсчитаем, сколько функций можно приблизить полиномами ранга $n-2$. Получаем, что число таких функций равно $2^{2^{n}-n-1}\left(2^{n}+1\right)$, то есть их меньше $2^{2^{n}}$. Отсюда следует, что $r_{2}^{(1)}(n) \geqslant n-1$.

Получаем, что $n-2 \leqslant r_{2}^{(q)}(n) \leqslant n-1$ для всех $n$, начиная с некоторого значения $n_{0}$ при $q \geqslant 2$, и $r_{2}^{(1)}(n)=n-1$.

Наконец, докажем шестое утверждение теоремы. Если $\delta=0$, то требуется построить полином, который не отличается от функции $f\left(x_{1}, \ldots, x_{n}\right)$ ни в одной точке, а значит, единственным подходящим полиномом будет полином, задающий функцию $f\left(x_{1}, \ldots, x_{n}\right)$, и $r_{k}^{\delta}(n)=n$.

Теорема 1 доказана.

Доказательство теоремь 2. Докажем первое утверждение. Если $\delta \geqslant(k-1) / k$, то полином с требуемым приближением можно построить аналогично тому, как это делалось в доказательстве утверждения 1 теоремы 1. Его длина будет равна 1. Полиномами меньшей длины не обойтись.

Следовательно, при $\delta \geqslant(k-1) / k$ справедливо равенство $l_{k}^{\delta}(n)=1$.

Докажем второе утверждение. Пусть $0<\delta<(k-1) / k$.

Получим верхнюю оценку. Определим множество $E, E \subseteq E_{k}^{n}$, по правилам:

(1) множество $E$ содержит нулевой набор, наборы, содержащие ровно $n-1$ нулей, наборы, содержащие ровно $n-2$ нулей, и т. д.

(2) каждый раз будем добавлять наборы одного типа, и только если наборы этого типа заканчиваются, будем переходить к другому типу;

(3) так будем действовать до тех пор, пока не наберем ровно $\left\lceil(1-\delta) k^{n}\right\rceil+(k-1)^{n}$ наборов;

(4) для выполнения условия леммы 6, может быть, будет нужно добавить еще не более $(k-1)^{n}-1$ наборов.

Пусть $|E|=l$. Заметим, что

$$
\left\lceil(1-\delta) k^{n}\right\rceil+(k-1)^{n} \leqslant l \leqslant\left\lceil(1-\delta) k^{n}\right\rceil+2(k-1)^{n}-1 .
$$

Обозначим через $X, X \subseteq X^{n}$, множество всех мономов, соответствующих наборам из множества $E$.

Рассмотрим функцию $f\left(x_{1}, \ldots, x_{n}\right)$ из $P_{k}^{n}$. Согласно лемме 6 , найдется полином $g^{\prime}\left(x_{1}, \ldots, x_{n}\right)$, в котором не более $l$ слагаемых, и значения функции $g^{\prime}\left(x_{1}, \ldots, x_{n}\right)$ совпадают со значениями функции $f\left(x_{1}, \ldots, x_{n}\right)$ на множестве $E$.

Пусть

$$
h(x)=x^{k-1}+x^{k-2}+\ldots+x+1 .
$$

Поскольку $h(x)=\left(x^{k}-1\right) /(x-1)$ и $x^{k}=x(\bmod k)$ при простых $k$, получаем, что $h(x)=1$ при $x \neq 1$. Несложно проверить, что $h(1)=0$. 
Пусть

$$
h_{n}\left(x_{1}, \ldots, x_{n}\right)=h\left(x_{1}\right) \ldots h\left(x_{n}\right) .
$$

Функция $h_{n}\left(x_{1}, \ldots, x_{n}\right)$ принимает значение 1 в $(k-1)^{n}$ точке и значение 0 в $k^{n}-(k-1)^{n}$ точках, ее полином имеет $k^{n}$ слагаемых, все коэффициенты в полиноме равны единице.

Оставим в полиноме $h_{n}\left(x_{1}, \ldots, x_{n}\right)$ только слагаемые, являющиеся мономами из множества $X$. Получим новый полином $h_{n}^{\prime}\left(x_{1}, \ldots, x_{n}\right)$ с $l$ слагаемыми.

Рассмотрим два случая.

Если в полиноме $g^{\prime}\left(x_{1}, \ldots, x_{n}\right)$ не более, чем $(k-1) l / k$ слагаемых, положим $g\left(x_{1}, \ldots, x_{n}\right)=g^{\prime}\left(x_{1}, \ldots, x_{n}\right)$. Различия между значениями функций $g\left(x_{1}, \ldots, x_{n}\right)$ и $f\left(x_{1}, \ldots, x_{n}\right)$ могут быть не более, чем в $k^{n}-\left\lceil(1-\delta) k^{n}\right\rceil-(k-1)^{n} \leqslant \delta k^{n}-(k-1)^{n} \leqslant \delta k^{n}$ точках.

В противном случае запишем полином $g^{\prime}\left(x_{1}, \ldots, x_{n}\right)$ в виде

$$
1 \cdot g_{1}^{\prime}\left(x_{1}, \ldots, x_{n}\right)+2 \cdot g_{2}^{\prime}\left(x_{1}, \ldots, x_{n}\right)+\ldots+(k-1) \cdot g_{k-1}^{\prime}\left(x_{1}, \ldots, x_{n}\right),
$$

где в полиномах $g_{s}^{\prime}\left(x_{1}, \ldots, x_{n}\right), s=1, \ldots, k-1$, все коэффициенты равны единице.

Выберем такое значение $s, 0 \leqslant s \leqslant k-1$, что длина полинома $g_{s}^{\prime}$ не меньше $l / k$. Такое $s$ всегда существует.

Пусть

$$
g\left(x_{1}, \ldots, x_{n}\right)=g^{\prime}\left(x_{1}, \ldots, x_{n}\right)-s h_{n}^{\prime}\left(x_{1}, \ldots, x_{n}\right) .
$$

При этом значения функции $g\left(x_{1}, \ldots, x_{n}\right)$ на множестве $E$ изменятся не более, чем в $(k-1)^{n}$ точке, то есть у функции $g\left(x_{1}, \ldots, x_{n}\right)$ различия с функцией $f\left(x_{1}, \ldots, x^{n}\right)$ могут быть не более, чем в $k^{n}-\left\lceil(1-\delta) k^{n}\right\rceil \leqslant \delta k^{n}$ точках. Длина полинома $g\left(x_{1}, \ldots, x_{n}\right)$ не больше $(k-1) l / k$.

В обоих случаях $g\left(x_{1}, \ldots, x_{n}\right)$ есть $\delta$-приближение функции $f\left(x_{1}, \ldots, x_{n}\right)$.

Длина полинома $g\left(x_{1}, \ldots, x_{n}\right)$ не больше $(k-1)\left(\left\lceil(1-\delta) k^{n}\right\rceil+2(k-1)^{n}-1\right) / k \leqslant$ $(k-1)\left((1-\delta) k^{n}+2(k-1)^{n}\right) / k$, то есть при $n \rightarrow \infty$

$$
l_{k}^{\delta}(n) \lesssim \frac{k-1}{k}(1-\delta) k^{n} .
$$

Получим верхнюю оценку. Рассмотрим функцию $h_{n}\left(x_{1}, \ldots, x_{n}\right)$ из $P_{k}^{n}$, построенную в доказательстве второго утверждения теоремы 2. Она принимает значение 1 в $(k-1)^{n}$ точке и ее полином содержит $k^{n}$ слагаемых.

Пусть дана функция $f\left(x_{1}, \ldots, x_{n}\right)$ из $P_{k}^{n}$. Если полином $f\left(x_{1}, \ldots, x_{n}\right)$ содержит не более $((k-1) / k) k^{n}$ слагаемых, положим полином $g\left(x_{1}, \ldots, x_{n}\right)$ равным полиному $f\left(x_{1}, \ldots, x_{n}\right)$.

В противном случае пусть полином $f\left(x_{1}, \ldots, x_{n}\right)$ имеет вид

$$
1 \cdot f_{1}\left(x_{1}, \ldots, x_{n}\right)+2 \cdot f_{2}\left(x_{1}, \ldots, x_{n}\right)+\ldots+(k-1) \cdot f_{k-1}\left(x_{1}, \ldots, x_{n}\right),
$$

где в каждом из полиномов $f_{s}\left(x_{1}, \ldots, x_{n}\right), s=1, \ldots, k-1$, все коэффициенты равны 1.

Выберем такое значение $s, 1 \leqslant s \leqslant k-1$, что длина полинома $f_{s}\left(x_{1}, \ldots, x_{n}\right)$ не меньше $(1 / k) k^{n}$. Такое значение $s$ всегда существует. Положим $g\left(x_{1}, \ldots, x_{n}\right)$, равным полиному $f\left(x_{1}, \ldots, x_{n}\right)-s h_{n}\left(x_{1}, \ldots, x_{n}\right)$.

В обоих случаях функция $g\left(x_{1}, \ldots, x_{n}\right)$ отличается от функции $f\left(x_{1}, \ldots, x_{n}\right)$ не более, чем в $(k-1)^{n}$ точке, что не больше $\delta_{n} k^{n}$. 
Полином $g\left(x_{1}, \ldots, x_{n}\right)$ содержит не более $(k-1 / k) k^{n}$ слагаемых. Отсюда следует, что $l_{k}^{\delta_{n}}(n) \leqslant(k-1 / k) k^{n}$. Получим нижнюю оценку. Пусть $\varepsilon>0, l(n)-$ положительные целые числа и $l(n) \leqslant((k-1) / k-\varepsilon) k^{n}$.

Подсчитаем число функций, которые можно приблизить полиномами длины, не превосходящей $l(n)$. Общее число полиномов длины, не большей $l(n)$, не превосходит $\sum_{j=0}^{l(n)}\left(\begin{array}{c}k^{n} \\ j\end{array}\right)(k-1)^{j}$.

Для каждого $n$ приближающие полиномы могут отличаться от приближаемых функций не более, чем в $\delta_{n} k^{n}$ точках. Тогда каждый из полиномов может быть приближением не более $\sum_{i=0}^{\left[\delta_{n} k^{n}\right]}\left(\begin{array}{c}k^{n} \\ i\end{array}\right)(k-1)^{i}$ функций. где $\left[\delta k^{n}\right]$ обозначает целую часть числа $\delta k^{n}$. Значит, полиномами длины $l(n)$ можно приблизить не более $\sum_{i=0}^{\left[\delta_{n} k^{n}\right]}\left(\begin{array}{c}k^{n} \\ i\end{array}\right)(k-1)^{i} \sum_{j=0}^{l(n)}\left(\begin{array}{c}k^{n} \\ j\end{array}\right)(k-1)^{j}$ функций.

По лемме 4 для всех $n$, начиная с некоторого значения $n_{0}$, выписанное произведение не больше $k^{\left(1-\varepsilon_{1}\right) k^{n}}$, где $\varepsilon_{1}, 0<\varepsilon_{1}<1$, - некоторая константа, то есть полиномов длины $l(n)$ не хватает для того, чтобы приблизить все $k^{k^{n}}$ функций от $n$ переменных при $n \geqslant n_{0}$. Отсюда получаем, что $l_{k}^{\delta_{n}}(n) \geqslant((k-1) / k-\varepsilon) k^{n}$ для любого $\varepsilon>0$ при всех $n$, начиная с некоторого значения $n_{0}=n_{0}(\varepsilon)$. Следовательно, $l_{k}^{\delta_{n}}(n) \sim((k-1) / k) k^{n}$ при $n \rightarrow \infty$.

Докажем четвертое утверждение. Пусть дана функция $f\left(x_{1}, \ldots, x_{n}\right)$ из $P_{2}^{n}$. Построим функцию $g\left(x_{1}, \ldots, x_{n}\right)$ из $P_{2}^{n}$ так же, как в доказательстве утверждения 3 теоремы 2. Она может отличаться от $f\left(x_{1}, \ldots, x_{n}\right)$ только на одном наборе $(0, \ldots, 0)$. Ее полином содержит не более $(1 / 2) 2^{n}$ слагаемых. Значит, $l_{2}^{(q)}(n) \leqslant(1 / 2) 2^{n}$.

Из предложения 1 и части 3 теоремы 2 получаем, что $l_{2}^{(q)}(n) \geqslant l_{2}^{\delta_{n}}(n) \geqslant(1 / 2-\varepsilon) 2^{n}$ для любого $\varepsilon>0$. Следовательно, $l_{2}^{(q)}(n) \sim(1 / 2) 2^{n}$ при $n \rightarrow \infty$.

Наконец, докажем пятое утверждение теоремы. Если $\delta=0$, то каждую функцию $f\left(x_{1}, \ldots, x_{n}\right)$ можно приблизить только задающим ее полиномом, и $l_{k}^{\delta}(n)=k^{n}$.

Теорема 2 доказана.

Автор благодарит В. Б. Алексеева за обсуждение результатов и ценные советы и замечания, которые улучшили текст статьи.

\section{Список литературы}

1. Джавадов Р. М., О сложности приближенного задания функций алгебры логики. Докл. АН СССР (1982) 265, №1, 24-27.

2. Яблонский С. В., Введение в дискретную математику. Наука, Москва, 1986.

Статья поступила 19.01.2007. 